\title{
REFLEXIONES SOBRE LA GESTIÓN DE LOS RECURSOS HÍDRICOS Y LA SALUD PÚBLICA EN EL PERÚ
}

\author{
Tania Burstein-Roda1,a
}

\begin{abstract}
RESUMEN
El presente artículo es un ejercicio para reflexionar sobre la situación actual del Perú en lo que se refiere al acceso a agua segura y sus consecuencias en la salud y sobre los desafíos que deberá atravesar el país para alcanzar la seguridad hídrica. Propone además algunos lineamientos para dirigirnos hacia la seguridad hídrica en el Perú. El artículo no pretende ser una fuente de referencia de datos estadísticos sobre la disponibilidad y acceso a los recursos hídricos en el Perú.
\end{abstract}

Palabras clave: Política hídrica; Gestión integrada de recursos hídricos; Planificación hídrica; Salud pública (fuente: DeCS BIREME).

\section{CONSIDERATIONS ABOUT MANAGEMENT OF WATER RESOURCES AND PUBLIC HEALTH IN PERU}

\begin{abstract}
This article is presented as an exercise to reflect on the current Peruvian situation in terms of access to safe water and its consequences on health. Additionally, it aims at pondering on the challenges that the country must face in order to achieve water security. It also proposes some guidelines to direct us toward water security in Peru. This article is not intended to be a source of reference of statistical data on the availability and access to water resources in Peru.
\end{abstract}

Keywords: Water policy; Conservation of water resources; Water resources planning; Public health (source: MeSH NLM).

\section{INTRODUCCIÓN}

Es imposible concebir la vida en nuestro planeta sin agua, pero ¿Qué tan conscientes estamos de las características con las que debe contar este recurso para asegurar la salud humana? ¿Qué tanto conocemos acerca de las condiciones de la oferta de agua y de las exigencias de la demanda para la salud pública? ¿Cómo puede el agua pasar de ser un recurso que da vida a uno que la destruye?

Las sociedades siempre se han instalado y desarrollado en las cercanías a fuentes de agua fresca, lo que les permitió producir sus alimentos a través de las actividades agropecuarias y volverse sedentarias. Todas las importantes culturas de la antigüedad han venerado al agua como fuente de vida y de transformación.

En la actualidad, problemas a nivel planetario como el calentamiento global y a nivel local como la sobreexplotación de los recursos hídricos debido a la presión demográfica, han puesto en la agenda mundial el estado crítico que ha alcanzado la humanidad en términos de disponibilidad y acceso al agua en cantidad y calidad suficientes.

«El Foro Económico Mundial en su informe anual sobre riesgos globales (2012), entendidos estos como eventos que, de ocurrir, pueden causar serios daños a países y sectores industriales, posicionó el 2015 a la crisis del agua como el riesgo de mayor preocupación [...] para el 2016 el riesgo de mayor significancia será la incapacidad de adaptarnos y mitigar el cambio climático, las armas de destrucción masiva ocuparán el segundo lugar, la crisis por el agua el tercero $[\ldots]{ }^{(1)}$

Si bien en las últimas décadas, las investigaciones en torno al agua se han centrado en los posibles modelos y estrategias de una gestión sostenible de los recursos hídricos, hoy ha tomado mucha relevancia el propósito de esta gestión: la seguridad hídrica.

\footnotetext{
Pontificia Universidad Católica del Perú. Lima, Perú.

a Licenciada en Geografía

Recibido: 25/04/2018 Aprobado: 06/06/2018 En línea: 06/07/2018
}

Citar como: Burstein-Roda T. Reflexiones sobre la gestión de los recursos hídricos y la salud pública en el Perú. Rev Peru Med Exp Salud Publica. 2018;35(2):297-303. doi: 10.17843/rpmesp.2018.352.3641. 
El estrecho vínculo entre agua y salud queda evidenciado en las diferentes definiciones que existen de seguridad hídrica. A continuación, se cita dos de las más aceptadas:

«La seguridad hídrica se define como la capacidad de una población para salvaguardar un acceso sostenible a cantidades adecuadas de agua de calidad aceptable para sostener los medios de vida, el bienestar humano y el desarrollo socioeconómico, para asegurar la protección contra enfermedades y desastres relacionados con el agua, y para preservar los ecosistemas en un clima de paz y estabilidad política» (2).

«La seguridad hídrica puede definirse como la provisión confiable de agua cuantitativa y cualitativamente aceptable para la salud, la producción de bienes y servicios y los medios de subsistencia, junto con un nivel aceptable de riesgos relacionados con el agua» ${ }^{(3)}$.

La seguridad hídrica aspira a la disponibilidad de agua y a la capacidad de acceso adecuados para todos los usos humanos (consumo, saneamiento, producción), para la conservación de los ecosistemas y para el mantenimiento de riesgos aceptables asociados al agua (desastres naturales, enfermedades, eventos extremos).

Esta condición puede alcanzarse a través de la implementación de políticas y estrategias derivadas de procesos de participación e interrelación equilibrados y planificados entre los diferentes sistemas y entre todos los actores y sectores de la sociedad y del gobierno, lo que hoy se conoce como gobernanza hídrica.

\section{EL ESTADO DE LA CUESTIÓN: LA SEGURIDAD HIIDRICA EN EL PERÚ}

Según la Ley de Recursos Hídricos de nuestro país (2009) «El agua es un recurso natural renovable, indispensable para la vida, vulnerable y estratégico para el desarrollo sostenible, el mantenimiento de los sistemas y ciclos naturales que la sustentan, y la seguridad de la Nación» (4). En la Política de Estado sobre los Recursos Hídricos (2012) el Estado se compromete a «[...] cuidar el agua como patrimonio de la Nación y como derecho fundamental de la persona humana al acceso al agua potable, imprescindible para la vida y el desarrollo humano de las actuales y futuras generaciones. Se debe usar el agua en armonía con el bien común, como un recurso natural renovable y vulnerable, e integrando valores sociales, culturales, económicos, políticos y ambientales» ${ }^{(5)}$. Para esto, entre otras medidas, el Estado dará prioridad al abastecimiento de agua en cantidad y calidad adecuadas para el consumo humano y la seguridad alimentaria; y asegurará el acceso universal al agua potable y saneamiento a todas las poblaciones urbanas y rurales.
El Perú está adherido a los Objetivos de Desarrollo Sostenible (ODS) de las Naciones Unidas (2015) ${ }^{(6)}$, cuyo ODS seis ${ }^{(7)}$ es el de garantizar la disponibilidad de agua y su gestión sostenible y el saneamiento para todos.

La Ley N³0588 (2017) incorpora el artículo 7-A a la Constitución Política del Perú que establece que «el Estado reconoce el derecho de toda persona a acceder de forma progresiva y universal al agua potable. El Estado garantiza este derecho priorizando el consumo humano sobre otros usos» ${ }^{(8)}$

Estos compromisos políticos y normativas son ciertamente relevantes en un país que se caracteriza por una aguda escasez de agua y altos índices de contaminación debido a la limitada accesibilidad y administración por la alta ineficiencia en la gestión de este recurso a pesar de contar con una extraordinaria oferta hídrica.

La Figura 1 muestra que la costa desértica del Perú concentra al $70 \%$ de la población y sólo cuenta con $2 \%$ de disponibilidad hídrica. Lima, la ciudad capital de alrededor de diez millones de habitantes es la segunda ciudad más grande del mundo ubicada en un desierto (la primera es El Cairo). La vertiente oriental, donde se ubica la cuenca amazónica, concentra el 97,4\% del recurso hídrico pero la población es poca, en su mayoría rural, con asentamientos dispersos por lo general.

A este problema de distribución demográfica, se suma la distribución del uso del agua. Como se muestra en la Figura 2, el $80 \%$ del recurso es utilizado en las actividades agrarias que se desarrollan sobre todo en la costa, lo que genera un grave problema de estrés hídrico, exacerbado por la selección de cultivos de agroexportación como el arroz en la costa norte o el espárrago en la costa central y sur que requieren de grandes cantidades de agua.

Esta fuerte demanda de agua para cultivos de exportación en zonas áridas no solo tiene como consecuencia la escasez del agua superficial si no que se ha venido sobreexplotando las reservas de agua subterránea, lo que tiene como consecuencia la intrusión de agua de mar en los acuíferos con su consecuente salinización y, por lo tanto, avance de la desertificación. A estos impactos se suma el uso de fertilizantes y pesticidas que contaminan las aguas y los suelos con nitratos.

Estas condiciones ponen en riesgo a la seguridad alimentaria entendida como el «acceso de la población a alimentos suficientes y de calidad, que les permita desarrollar una vida activa y saludable dentro de una concepción de desarrollo humano integral» (9) ya que la disponibilidad de alimentos depende en gran medida por la disponibilidad de agua en cantidad y calidad adecuadas.

Esto se agrava en países como el Perú, con un incesante crecimiento demográfico que se concentra sobre todo en 
Vertiente del Pacífico $21,8 \%$ del territorio $70 \%$ de la población $2 \%$ de la disponibilidad hídrica $80 \%$ del PBI

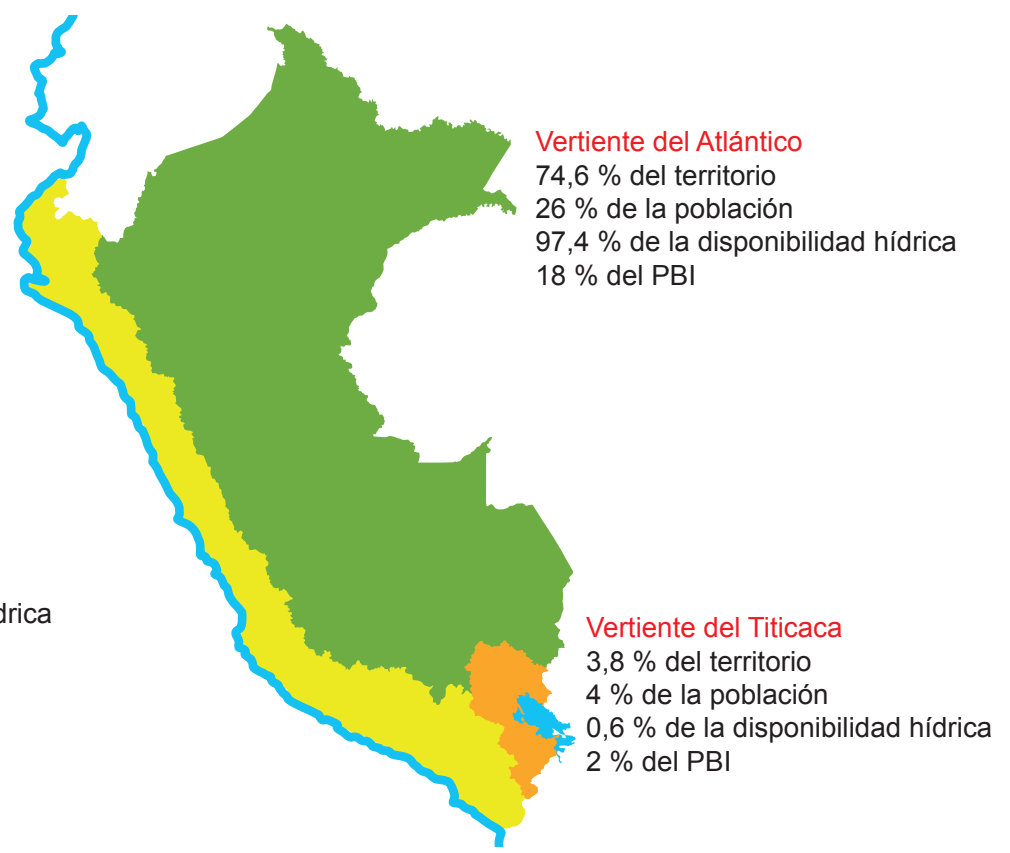

Figura 1. Disponibilidad hídrica y distribución demográfica en el Perú

Fuentes: ANA,MINAM, MEF. Elaboración propia

ciudades, las altas tasas de urbanización se traducen en una mayor demanda de alimentos, de agua potable y de servicios de saneamiento básico. La Figura 3 muestra el importante proceso de urbanización en Perú.

La escasez de recursos hídricos, la mala calidad del agua y el saneamiento inadecuado influyen negativamente en la seguridad alimentaria ${ }^{(7)}$. Según el último informe de UNICEF PERÚ, el 26,6 \% de los hogares no dispone de red pública de agua y, en algunas zonas de la Amazonía esta cifra alcanza $75 \%$. El 35,8 \% carece de red pública de desagüe, situación que pone en riesgo la salud de niñas y niños ${ }^{(10)}$.

Esta información se corrobora con los datos de la Encuesta Demográfica y de Salud Familiar 2016 (ENDES), que

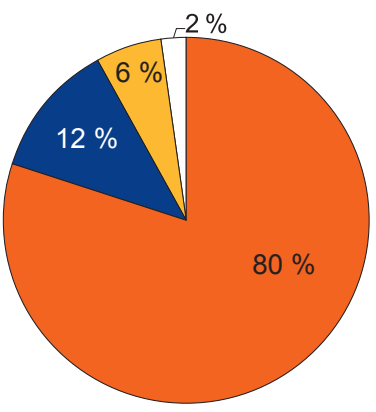

$\square$ Agrícola

— Poblacional $\square$ Industrial

$\square$ Minero

Figura 2. Usos del Agua en el Perú

Fuente: Autoridad Nacional del Agua reporta que $73,4 \%$ de personas obtienen agua para beber y $64,2 \%$ tienen servicios sanitarios con conexión a la red pública dentro de la vivienda. Dichos porcentajes mejoraron respecto al $2012(71,7 \%$ y 54,4\%, respectivamente). En el $2016,77,6 \%$ y $61,5 \%$ de la población que reside en áreas urbanas y rurales, respectivamente obtenía agua para beber de la red pública dentro la vivienda, mientras que $80,8 \%$ en área urbana y $16,2 \%$ en área rural tenían servicios sanitarios dentro de la vivienda con conexión a la red pública.

Si bien se ha avanzado en la dotación de estos servicios, aún existen deficiencias sobre todo en las áreas rurales y en las zonas urbano marginales ocupadas por poblaciones con mayores niveles de vulnerabilidad por las condiciones de pobreza en las que viven.

Existe una relación directa entre la ausencia de servicios de agua potable y saneamiento y el incremento de la prevalencia de enfermedades diarreicas, en especial entre niñas y niños menores de cinco años, lo que vulnera al mismo tiempo su estado nutricional (11); en el Perú, la prevalencia de enfermedades diarreicas agudas (EDA) genera desnutrición infantil y es causa de mortalidad.

Según la ENDES $2016^{(12)}$, la prevalencia de diarrea entre niñas y niños menores de cinco años alcanzó el 11,5\%, un porcentaje menor al encontrado en la encuesta 2012 $(12,3 \%)$. Se encontraron diferencias poco significativas por sexo (12,1 \% en niños y $10,9 \%$ en niñas) o área de residencia (11,4\% urbana y $11,8 \%$ rural); lo que podría indicar un cierto progreso en las campañas de acceso a servicios de salud. 


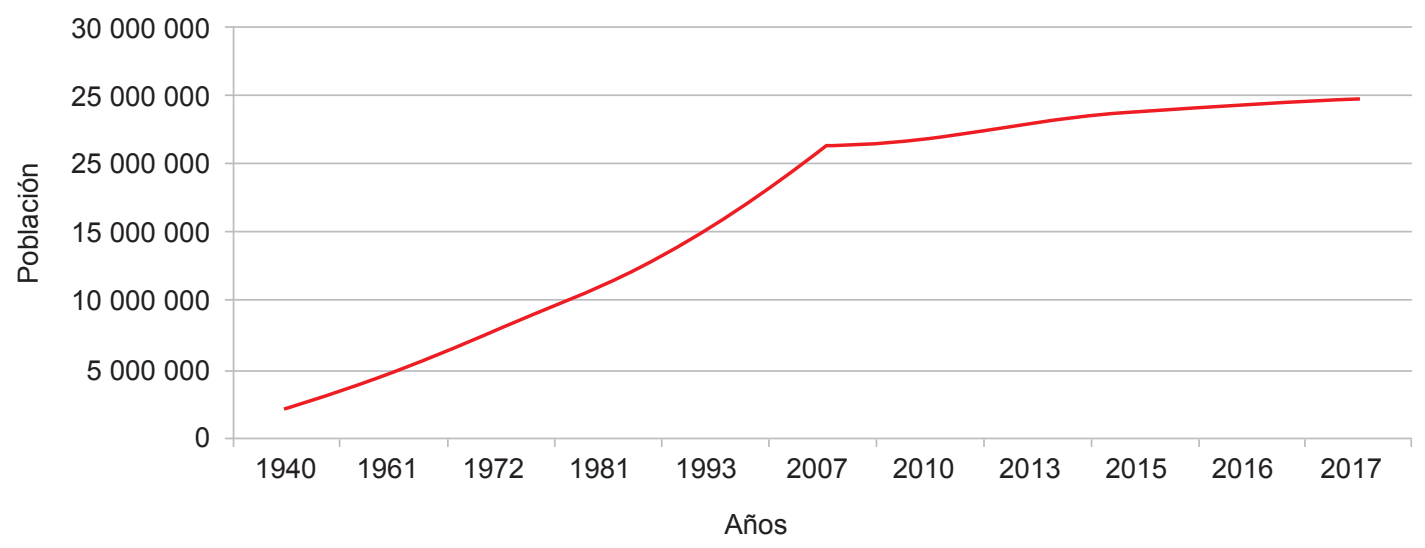

Figura 3. Evolución de la población urbana en el Perú 1940-2017

Fuente: Censos de Población y Vivienda y proyecciones demográficas del INEI.

Por región, se encontró una mayor prevalencia entre las niñas y niños residentes en Ucayali $(20,1 \%)$ y Ayacucho $(20,0 \%)$; mientras que, la prevalencia fue menor en Moquegua (7,6 \%), la provincia de Lima $(8,9 \%)$ e lca $(9,1 \%)$ lo que concuerda con las brechas en los servicios de agua potable y saneamiento entre regiones.

También se obtuvo diferencias dependiendo de las características de la vivienda, siendo mayor en niñas y niños que habitan viviendas cuya fuente de agua para beber es no mejorada $(13,1 \%)$ en comparación con la mejorada (11,3\%); asimismo, se observó una mayor prevalencia en niñas y niños que habitan viviendas con servicio sanitario no mejorado o compartido (12,0\%) en comparación con el mejorado y no compartido (11,2\%).

Respecto a la asociación entre diarrea y fuente de agua para beber, el porcentaje de diarrea en niñas y niños fue menor cuando consumen agua tratada con cloro residual (11,0\%). Asimismo, este porcentaje fue similar cuando consumen agua hervida proveniente de otra fuente $(11,2 \%)$ o de la red pública (11,4\%); en tanto, fue mayor entre aquellas y aquellos que consumen agua sin tratamiento (14,2\%).

La estrecha relación entre cobertura de agua potable y desagüe, la prevalencia de EDA y la desnutrición crónica se comprueba con los resultados obtenidos por esta misma encuesta (ENDES 2016) para la desnutrición crónica infantil. A nivel nacional, la desnutrición crónica afectó al $13,1 \%$ de niñas y niños menores de cinco años, esta situación reflejaría una tendencia decreciente al observar el $2012(18,1 \%)$.

Por sexo, la desnutrición crónica presentó una diferencia de 1,8 puntos porcentuales $(14,0 \%$ en niños y $12,2 \%$ en niñas). Según área de residencia, la desnutrición crónica afectó en mayor proporción a niñas y niños del área rural $(26,5 \%)$, es decir, 18,6 puntos porcentuales más que en el área urbana $(7,9 \%)$.
Por tratamiento del agua, las niñas y niños que consumieron agua sin tratamiento presentaron mayor porcentaje de desnutrición crónica $(26,0 \%)$, seguido de quienes consumieron agua hervida proveniente de otra fuente distinta a la red pública (20,2\%), en comparación con los que consumen de agua hervida que procede de red pública $(13,5 \%)$ o con cloro residual $(5,7 \%)$.

Al 2018, el Perú, a pesar de contar con un marco político y normativo orientado y comprometido a una gestión sostenible de los recursos hídricos, aún tiene enormes brechas en el abastecimiento de agua en cantidad y calidad necesarias para la cobertura de servicios básicos y el desarrollo de las actividades productivas y de conservación de ecosistemas.

La escasez de agua que pone en riesgo la seguridad alimentaria, sumada a la mala calidad del recurso que permite la prevalencia de enfermedades diarreicas, condiciona a la población a padecer importantes niveles de desnutrición o malnutrición que la predisponen a desarrollar enfermedades y a no lograr el desarrollo cognitivo adecuado. Estos efectos se prolongan para toda la vida aumentando el riesgo de desarrollar enfermedades crónicas y a tener bajos rendimientos en los estudios y en la vida laboral.

A la situación actual se suma que esas afecciones y otros resultados sanitarios se verán cada vez más afectados por la aceleración del cambio climático debido a sus efectos adversos en la producción de alimentos, la disponibilidad de agua y la dinámica de las poblaciones de vectores y agentes patógenos. La mayor parte de las enfermedades existentes son ocasionadas por determinadas características climáticas (temperaturas muy altas o muy bajas, humedad atmosférica muy alta o muy baja, intensidad de los vientos, intensidad y frecuencia de las lluvias, etc.), por determinadas características en el recurso hídrico (escasez, contaminación) o por la combinación de ambas (inundaciones, huracanes, sequías, etc.). 
La capacidad productiva del recurso hídrico es ilimitada si su gestión es sostenible. Además del uso compartido, esta gestión debe estar orientada a minimizar la fuerza destructiva del agua. La mayor parte de desastres naturales deben sus efectos devastadores al agua, ya sea al exceso o a la ausencia de esta.

Los desastres naturales relacionados con el exceso de agua son las inundaciones, los huaicos y movimientos de grandes porciones de terreno y hielo (deslizamientos, avalanchas) y las tormentas/huracanes que generan tsunamis. Los relacionados con su ausencia son las sequías y los incendios forestales.

La ocurrencia de sequías e inundaciones, ponen en grave riesgo a la economía del país que se basa en actividades que dependen directamente del clima, como la agricultura y el turismo. Esto es especialmente preocupante dado que, un gran porcentaje de los peruanos que vive bajo la línea de la pobreza se dedica a la agricultura como su principal actividad de subsistencia.

El Perú es uno de los países más afectados por fenómenos hidrometeorológicos relacionados con el Fenómeno El Niño (FEN) y las perturbaciones océano atmosféricas generadas en el Océano Pacífico ecuatorial tropical (PNUD-MINAM, 2009). El mayor porcentaje (72 \%) de las emergencias se relacionan a fenómenos de origen hidrometeorológicos (sequías, fuertes lluvias, inundaciones, heladas, granizadas) y han registrado un crecimiento de más de seis veces desde 1997 al 2006. Los escenarios de cambio climático generados para el Norte del Perú indican la probabilidad de una intensificación del FEN.

En cuanto a las posibles consecuencias del cambio climático sobre la salud pública, la OMS ${ }^{(13)}$ se ha pronunciado de la siguiente manera:

- Según se prevé, entre 2030 y 2050 el cambio climático causará unas 250000 defunciones adicionales cada año, debido a la malnutrición, el paludismo, la diarrea y el estrés calórico ${ }^{(14)}$.

- Muchas de las enfermedades más mortíferas, como las diarreas, la malnutrición, la malaria y el dengue, son muy sensibles al clima y es de prever que se agravarán con el cambio climático ${ }^{(15)}$.

- Las zonas con malas infraestructuras sanitarias, que se hallan en su mayoría en los países en desarrollo, serán las menos capacitadas para prepararse ante esos cambios y responder a ellos si no reciben ayuda.

La OPS ${ }^{(16)}$ agrega:

- Se prevé que, en los próximos decenios, aumentará significativamente el número de personas expuestas al alcance geográfico y la incidencia de enfermedades de transmisión vectorial, zoonóticas y de transmisión alimentaria e hídrica.
- Los fenómenos meteorológicos extremos aumentarán su frecuencia e intensidad sugiriendo que los efectos asociados con la salud podrían aumentar si no se adoptan más medidas de prevención.

- El cambio climático puede afectar la salud de la población debido a trastornos económicos provocados por el clima y el deterioro ambiental, así como a retrocesos en el desarrollo resultantes de daños causados en la infraestructura de salud pública y en los medios de vida por fenómenos meteorológicos extremos.

En el sector salud a nivel nacional, se cuenta con poca evidencia para poder pronosticar qué pasará exactamente. Sin embargo, las investigaciones realizadas acerca del Fenómeno del Niño y su relación con la incidencia de algunas enfermedades ayudan a predecir los efectos en la salud en un clima más cálido.

Por lo tanto, según las predicciones a nivel global y regional, y tomando en cuenta los efectos del fenómeno de El Niño, se infiere que aumentarán significativamente:

- Los casos de enfermedades transmitidas por el agua.

- Los casos de enfermedades de transmisión vectorial y zoonótico.

- Los casos de enfermedades transmitidas por alimentos.

- Los casos de lesión y muerte por eventos climáticos extremos.

- Los casos de enfermedades mentales por traumas posteriores a los eventos climáticos extremos.

- La desnutrición y malnutrición.

El panorama para alcanzar la seguridad hídrica se torna sombrío y los desafíos son grandes, pero no imposibles si se involucra a todos los actores estratégicos, se toman las decisiones acertadas y se adapta de manera eficiente a nuestras diversas realidades las estrategias propuestas por los expertos internacionales para la gestión de los recursos hídricos.

\section{DESAFÍOS PARA LA SEGURIDAD HÍDRICA: LA GESTIÓN INTEGRADA DE LOS RECURSOS HÍDRICOS A TRAVÉS DE LA GOBERNANZA HIIDRICA}

A continuación, y para continuar con esta reflexión, se toma como referencia el siguiente argumento: «Un mundo con seguridad hídrica aprovecha la capacidad productiva del agua y minimiza su fuerza destructiva. Es un mundo donde todas las personas tienen suficiente agua segura y a un precio accesible para llevar una vida limpia, sana y productiva. Es un mundo donde las comunidades están protegidas de inundaciones, sequías, desprendimientos de tierra, la erosión y las enfermedades transmitidas por el agua» (17). 
La gestión del agua en nuestro país está sectorizada, sin canales efectivos para integrar de manera transectorial las diferentes políticas y planes relacionados con ella.

El primer paso es enfocarse en la complejidad de su gestión y en su naturaleza multifuncional para las sociedades y el medio ambiente ${ }^{(18)}$. La gestión del agua involucra a actores no gubernamentales y a todos los sectores y niveles del gobierno, atraviesa diversas disciplinas técnicas y científicas, requiere de interrelaciones entre usos y usuarios, comprende a nuevos usos y a usos tradicionales consuetudinarios y no se puede desprender de su componente político.

Esta complejidad requiere de formas alternativas e innovadoras de organización para su gestión ${ }^{(18)}$, pasar de la tradicional administración jerárquica del Estado a formas más horizontales e inclusivas en la toma de decisiones, de enfoques tecnicistas sectoriales a enfoques transversales e integrados. La innovación para evaluar, entender y desarrollar soluciones toma un rol relevante.

La gestión integrada de los recursos hídricos (GIRH), tomando como espacio de aplicación a la cuenca hidrográfica, considera esta complejidad y permite una administración y distribución del recurso hídrico en forma concertada y eficientes. La GIRH es la nueva estrategia planteada a nivel mundial para alcanzar la seguridad hídrica, alimentaria y energética. «Es un proceso que promueve el desarrollo y manejo coordinado del agua, la tierra y otros recursos relacionados, con el fin de maximizar el bienestar económico y social resultante de manera equitativa, sin comprometer la sostenibilidad de los ecosistemas vitales» ${ }^{(19)}$

El Perú se ha comprometido a implementar la GIRH en la Política de Estado sobre Recursos Hídricos y la ha declarado de interés nacional y necesidad pública en el Título I, disposiciones generales, Artículo 3 de la Ley de Recursos Hídricos, Ley No. 29338. La puesta en práctica de esta estrategia es además una de las metas del ODS 6 de las Naciones Unidas al cual está adherido nuestro país.

En este contexto, la participación de la sociedad civil y la empresa privada toma relevancia en la toma de decisiones y en la implementación de políticas públicas. La sociedad toma un rol central en el diseño de los instrumentos diseñados para superar los desafíos que presenta la seguridad hídrica.

Este enfoque entiende que la lucha contra la desnutrición y la prevalencia de enfermedades relacionadas con la falta de agua o con agua de mala calidad, no es solo tarea del sector salud, sino que requiere de estrategias construidas de abajo hacia arriba, desde los que sufrirán de la escasez o consumirán agua contaminada hacia actores estratégicos académicos y privados, que pueden entregar conocimiento o financiamiento y hasta los sectores públicos que tienen la responsabilidad del abastecimiento de agua en cantidad y calidad suficientes para asegurar la salud de la población. Esta forma de construir las soluciones a los problemas hídricos no solo permitirá contar con respuestas acertadas y eficientes, sino que resultará en estrategias que serán entendidas, aceptadas y de las que se apropiará la sociedad, la misma que deberá implementarlas.

En este punto, la GIRH requiere de un sistema de organizaciones y procesos con la suficiente legitimidad, eficiencia y sostenibilidad para su diseño e implementación. Surge entonces el concepto de Gobernanza Hídrica como marco político e institucional para el desarrollo de la GIRH. El concepto de gobernanza se focaliza en las formas y tipos de interrelación entre la sociedad civil y el Estado e inicia una serie de análisis sobre modelos y formas de gobernanza tomado en cuenta la estructura de las organizaciones (formal, informal, mixtas) y sus procesos (bottom up, top-down) ${ }^{(1)}$.

Citando a la Asociación Mundial para el Agua (GWP, por sus siglas en inglés) la gobernanza hídrica «hace referencia a la implementación efectiva y socialmente aceptada de responsabilidad y regulación y es, por lo tanto, de una naturaleza extremadamente política [...] es un concepto más inclusivo que el gobierno per se ya que abarca la relación entre una sociedad y su gobierno [...] abarca leyes, normativas e instituciones pero también enlaza las políticas públicas con las actividades locales y redes de influencia constituidas por las fuerzas del mercado internacional, del sector privado y de la sociedad civil [...]» (20).

«La gobernanza del agua hace referencia al conjunto de sistemas políticos, sociales, económicos y administrativos, implementados para el desarrollo y gestión de los recursos hídricos y la provisión de servicios de saneamiento en los diferentes niveles de la sociedad ${ }^{(20)}$.

La gobernanza hídrica supone entonces la existencia de políticas públicas claras, un marco jurídico adecuado, así como sistemas de participación social e instituciones apropiadas y con las capacidades necesarias. Supone también la coordinación entre los diversos actores y en los diferentes ámbitos territoriales, un concepto que la Organización para la Cooperación y el Desarrollo Económico (OCDE) traduce como «gobernanza multinivel» (1).

En el Perú, el marco normativo está dado, hace falta la voluntad política para generar las condiciones económicas y los mecanismos de interacción transectorial y de participación de los actores estratégicos no gubernamentales para constituir la gobernanza hídrica que permitirá la construcción de la GIRH en todas las cuencas hidrográficas de nuestro país. 


\section{REFERENCIAS BIBLIOGRÁFICAS}

1. Martínez-Austria P. La seguridad hídrica. En: Meller P, Broschek U, Velázquez L, Solís de Ovando C, editores. Desafíos del agua para la región latinoamericana. Santiago de Chile: Fundación Chile; 2017.p. 30-43.

2. World Water Assessment Programme. Agua para todos, agua para la vida. Informe de las Naciones Unidas sobre el Desarrollo de los Recursos Hídricos en el Mundo. Resumen Ejecutivo Paris: UNESCO; 2003.

3. Sadoff, C, Muller, M. La gestión del agua, la seguridad hídrica y la adaptación al cambio climático: efectos anticipados y respuestas esenciales. Estocolmo: Global Water Partnership; 2010. TEC Background Papers, 14.

4. Congreso de la República del Perú, Ley de Recursos Hídricos. Ley No. 29338 (30 marzo 2009). El Peruano. Normas Legales. 2009; XXVI(10562):393473-86.

5. Acuerdo Nacional. Trigésima Tercera Política de Estado. Política de Estado sobre los Recursos Hídricos. Lima, 2012.

6. Naciones Unidas [Internet] Objetivos de desarrollo sostenible; 17 objetivos para transformar nuestro mundo. Nueva York; 2017 (citado el 15 de enero de 2018). Disponible en: http://www.un.org/ sustainabledevelopment/es/objetivos-dedesarrollo-sostenible/

7. Naciones Unidas [Internet] Objetivos de desarrollo sostenible; 17 objetivos para transformar nuestro mundo. Objetivo 6: Garantizar la disponibilidad de agua y su gestión sostenible y el saneamiento para todos. Nueva York; 2017 (citado el 15 de enero de 2018). Disponible en: http:// www.un.org/sustainabledevelopment/es/ water-and-sanitation/

8. Congreso de la República del Perú. Ley $\mathrm{N}^{\circ} 30588$ (21 junio 2017). Ley de reforma constitucional que reconoce el derecho de acceso al agua como derecho constitucional, El Peruano. Normas Legales. 2017;XXXIV (14117):4.

9. Acuerdo Nacional. Décimo quinta política de Estado. Promoción de la seguridad alimentaria y nutrición. Lima; 2004 [citado el 20 de enero de 2018]. Disponible en: https://www. acuerdonacional.pe/wpcontent/ uploads/2004/01/15-Inf-matriz-2004plus.pdf.

10. UNICEF [Internet] UNICEF PERÚ 2017-2021. 10.5 Millones de Motivos para Seguir Trabajando. Lima, 2017 [citado el 20 de enero de 2018]. Disponible en: https://www.unicef.org/peru/spanish/10.5_millones_de_motivos_para_seguir_trabajando_-_Web.pdf

11. Oblitas de Ruiz L. CEPAL, Servicios de Agua Potable y Saneamiento en el Perú: beneficios potenciales y determinantes de éxito. Santiago de Chile: CEPAL; 2010. Documentos de Proyectos e Investigación, 355.

12. Instituto Nacional de Estadística e Informática. Perú: Encuesta Demográfica y de Salud Familiar 2016; nacional y regional. Lima; INEI; 2017.

13. Organización Mundial de la Salud. Cambio climático y salud. Ginebra: OMS; 2014. Nota Descriptiva, 266. (citado el 20 de enero de 2018) Disponible en: http:// www.who.int/mediacentre/factsheets/ fs266/es/
14. World Nealth Organization. Global health risks: mortality and burden of disease attributable to selected major risks. Geneva: WHO; 2009.

15. Hales S. et al. Potential effect of population and climate changes on global distribution of dengue fever: an empirical model. The Lancet, 2002,360(9336):830-4.

16. Organización Panamericana de la Salud. Proteger la salud frente al cambio climático: evaluación de la vulnerabilidad y la adaptación. Washington DC. OPS; 2012.

17. Global Water Partenership. La estrategia de GWP hacia el 2020: un mundo con seguridad hídrica. Estocolmo: GWP; [s.f.]

18. Hakan T, Water governance: trends and needs for new capacity development. Water Policy. 2007; 9 Supplement 2:19-30.

19. Global Water Partnership. ¿Qué es la GIRH?, Estocolmo: GWP; 2011 (citado el 15 de enero de 2018). Disponible en:https://www.gwp.org/es/GWPSud-America/ACERCA/por-que/ PRINCIPALES-DESAFIOS/Que-es-laGIRH/

20. Rogers P, Hall A. Gobernabilidad efectiva del agua. Estocolmo: GWP; 2006. TEC Background Papers, 7.

Correspondencia: Tania Burstein Roda Dirección: Calle Cabo Blanco 160, dpto. 2, San Isidro. Lima, Perú

Teléfono: (511) 992348267.

Correo electrónico: taniaburstein@yahoo.com 